\title{
Análisis documental de las implicaciones de la tecnología como medio de innovación en la toma de decisiones empresariales
}

\author{
Ivonne Janette Silva Almanza ${ }^{1}$, Margarita Amada Ponce González ${ }^{2}$ y María Guadalupe \\ García Garza ${ }^{3}$ \\ ${ }^{1}$ Universidad Autónoma de Nuevo León, ivonne.silvaalm@uanl.edu.mx, Av.Universidad S/N Cd. \\ Universitaria, San Nicolás de los Garza, N. L., +52 8111687476. \\ ${ }^{2}$ Universidad Autónoma de Nuevo León, margarita.poncegn@uanl.edu.mx, Av. Universidad S/N Cd. \\ Universitaria, San Nicolás de los Garza, N. L., +52 8110693153. \\ ${ }^{3}$ Universidad Autónoma de Nuevo León, maria.garciagrza@uanl.edu.mx, Av. Universidad S/N Cd. \\ Universitaria, San Nicolás de los Garza, N. L., +52 8120029295.
}

Información del artículo revisado por pares
Fecha de aceptación: junio-2021
Fecha de publicación en línea: diciembre-2021
DOI: https://doi.org/10.29105/vtga7.2-26

\section{Resumen}

Uno de los ejes centrales de toda organización es la toma de decisiones, cuya base estructural es la información, misma que si es debidamente gestionada a través de herramientas tecnológicas pueden brindar de certeza a su usuario, que a su vez asegura decisiones que pueden brindar de ventaja competitiva a la organización que la gestione. Sin embargo, la tecnología es un factor que se mantiene en constante dinamismo, y como consecuencia, se debe atender las implicaciones que ésta tiene en la toma de decisiones dentro de las organizaciones.

Palabras clave: Toma de Decisiones, Tecnología, Ventaja Competitiva, Información, Innovación Tecnológica.

\section{INTRODUCCIÓN}

Una de las acciones principales de todo buen administrador es la optimización, y ésta no es más que una manera de tomar una decisión de entre varias alternativas posibles, el contexto en el cuál una persona enfrenta un futuro, cuyas probabilidades de éxito se incrementan entre más información se tenga al respecto, no siempre se encuentra claro, pues en muchas ocasiones la falta de datos, información,

\begin{abstract}
One of the central axes of any organization is decision-making, whose structural basis is information, which if properly managed through technological tools can provide certainty to its user, which in turn ensures decisions that can provide competitive advantage to the organization that manages it. However, technology is a factor that remains in constant dynamism, and as a consequence, the implications that it has on decisionmaking within organizations must be addressed.
\end{abstract}

Keywords: Decision Making, Technology, Competitive Advantage, Information, Technological Innovation.

JEL:M15, O3, O32.

pronósticos, estadísticas, casos similares, limitan la certidumbre e incrementan el riesgo de no tomar una buena decisión, lo que incurre en la pérdida de oportunidades.

Peñaloza Palomeque (2010) comenta que la decisión en condiciones de incertidumbre dependerá netamente de la personalidad del decisor, como consecuencia su riesgo es alto. Es decir, las decisiones se clasifican en aquellas que se dan en condiciones de certeza, 
en condiciones de riesgo y en condiciones de incertidumbre, y todas estarán determinadas por el grado de información y conocimiento con el que se cuente.

Tanto la información como el conocimiento son en la actualidad, considerados como activos intangibles, que ganan cada día mayor terreno en materia de planeación estratégica, debido a que las empresas compiten en entornos más dinámicos, que requieren de adecuados mecanismos tecnológicos, además de la virtud de gestionar de manera eficiente sus competencias internas. Cohen (2002), describe cómo las organizaciones utilizan la información, desde el control de inventarios -materia prima-, horas máquina disponibles, tiempo de entrega de productos, hasta el uso de información por parte de los clientes, con la finalidad de reducir costos.

Todo esto para llevar a cabo una toma de decisiones más efectivas para la organización, que no se logran cuando existe un panorama de alta presión, con información desordenada $\mathrm{y}$ en un contexto de incertidumbre e incluso riesgo.

Hoy por hoy no puede exponerse el concepto de organización, sin tomar en cuenta el valor del capital intelectual (CI), Juma (2004), señala que el CI ha llegado a ser un factor esencial para la sobrevivencia de las organizaciones y el desarrollo actual de las empresas. Por lo que se ha vuelto indispensable disponer, entre otras cosas, de una estrategia, de un plan de objetivos, así como de un sistema de control de gestión de la información, en donde la tecnología se ha involucrado de manera activa, formando parte protagonista en estas tareas y facilitando la toma de decisiones.

Reyes Ortiz (2014) señala, que la tecnología de la información transforma la manera en que se producen y distribuyen los bienes y servicios. Debido a que se vive en una era del conocimiento, en donde las empresas deben incentivar medios que posicionen al ser humano en el centro, de modo que se potencie el conocimiento basado en información.

Lo anterior orienta a diseñar planes y métodos para identificar los medios a través de los cuáles se pueda optimizarse la toma de decisiones a través de una adecuada gestión de la información sustentada por la participación de los sistemas tecnológicos, y así lograr perfeccionar la curva de aprendizaje, a través de la propia retroalimentación del sistema y alcanzando una verdadera mejora continua.

Molina (2015) señala que, "La expresión trabajador del conocimiento- se debe a Peter Drucker, creador de la Dirección por Objetivos en los años 50's, refiriéndose a trabajadores que se dedicaban a gestionar el nuevo objeto de la economía de la sociedad pos capitalista: La información."

Como consecuencia, Drucker ya reconocía desde los 50's a las personas que poseían un saber específico utilizado para optimizar la toma de decisiones, requiriendo de una organización donde pudiera integrar la información disponible y los conocimientos a un todo mayor, y es en esta fusión donde la tecnología forma parte activa del resultado que es una toma de decisiones óptima y confiable.

Otros autores que participaron en el fortalecimiento del estudio de la gestión del conocimiento en las organizaciones fueron Thomas H. Davenport y Lawrence Prusak (1998), quienes consideraban al conocimiento como: "Una mezcla fluida de experiencia formulada, valores, información contextual y visión experta".

Por lo que se hace importante profundizar en el estudio de las implicaciones que tiene la tecnología al tomar un papel de innovación en la toma de decisiones empresariales, dentro de un mercado globalizado que exige que los miembros comerciales cuenten con el mayor grado de información.

Situación que combinada con los conocimientos tácticos y explícito, garanticen una toma de decisiones más efectiva, y que no se vea obstaculizada por escenarios de riesgo o de incertidumbre como consecuencia de la falta de equipación tecnológica para gestionar la información.

\section{MARCO TEÓRICO DE LA TOMA DE DECISIONES EMPRESARIALES.}

La Información y el conocimiento son factores clave en materia de innovación y competitividad de las organizaciones, de modo que las empresas buscan formas de 
crear, transformar, automatizar y sistematizar dicha experiencia. Nagles García (2018) comenta que la información que posee una organización es una fuente de ventaja competitiva sostenible, mediante el despliegue de una efectiva estrategia de toma de decisiones se logran acciones innovadoras para optimizar los recursos y capacidades de la empresa.

\subsection{La toma de decisiones en las empresas.} La toma de decisiones comprende la elección de entre dos o más alternativas que conducen a un fin deseado, este tema ha formado parte central de varios estudios en materia de gestión empresarial, pues de ella depende la distribución de actividades, responsabilidades e incluso la determinación de autoridad, jerarquías y responsabilidades, esto representa incluso la distribución y asignación de la capacidad y autoridad para la toma de decisiones, entre mayor sea el nivel jerárquico mayor responsabilidad y peso existirá en sus decisiones.

Daft (2010) define a la toma de decisiones como el proceso de identificar y resolver los problemas en la organización cuando se consideran cursos alternativos de acción y se selecciona e implanta una opción.

El concepto no puede limitarse a la resolución de problemas, ya que incluso la selección de una alternativa de proyecto representa una toma de decisiones importantes y no implica una situación problemática, sin embargo, es cierto que esta acción va a estar condicionada por recursos materiales, financieros, de recurso humano, lo que significa que son procesos que implican una inversión de capital que forma parte del patrimonio de la empresa, y que mientras más alejado se encuentre el resultado de la eficacia y eficiencia administrativa, peor serán considerados la capacidad de quien toma dichas decisiones.

El primer paso es la descripción de la situación, en la que se debe elaborar una síntesis del problema y sus principales componentes, así como los responsables del mismo, en este caso se sintetizan los aspectos principales del panorama a resolver, las variables que intervienen en el análisis, alternativas y enfoques; de manera secuencial se debe dar una estructuración al problema, y para ello se debe identificar y describir los aspectos principales de la toma de decisión, los principales actores o agentes involucrados, y la relación entre ellos.

A continuación, se presenta la figura No. 1 que resume los pasos de la metodología señalada por Castillo Hernández (2018) para la toma de decisiones y que deben ser ajustados y modificados según las características específicas del escenario que se esté analizando:

Figura 1. Metodología para el análisis de decisiones.

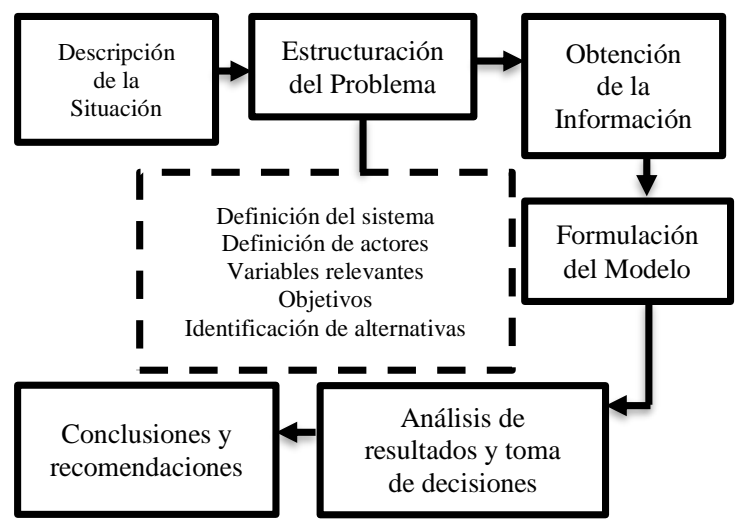

Fuente: (Castillo Hernández, 2018)

Para poder así explicitar las alternativas de decisión del problema y precisar de manera clara el escenario que se pretende analizar, sus objetivos y posibles limitaciones de análisis. De modo que el siguiente paso es la obtención de la información y datos necesarios para el análisis del escenario, tanto de las variables independientes como de la que se encuentra sujeta a los efectos de éstas.

$\mathrm{Se}$ formula y construye un modelo en donde se determine el proceso de decisión, las variables relevantes, criterios de decisión y alternativas a evaluar, y finalmente se obtienen y analizan los resultados, para ello se seleccionan las mejores alternativas, a través de identificar debilidades, fortalezas, oportunidades y amenazas de cada una de ellas.

Ahora bien, Hodge et al. (1998) señalan que "los miembros de la organización pueden pretender, con las mejores intenciones, tomar 
decisiones lógicas y razonables, pero las condiciones de la vida real establecen límites y reducen su capacidad para actuar de manera racional".

Esto ha generado que cada organización maneje modelos y sistemas de toma de decisiones diseñadas según su propio contexto, pero será la realidad de cada escenario la que determinará la mejor metodología para la toma de decisiones en la organización, por lo que la tecnología termina siendo un medio a través del cual se puede facilitar la gestión de la información, sin embargo, será el talento humano el que conjugue todos estos elementos para tomar la decisión más óptima.

\subsection{Datos, información y conocimiento en las organizaciones}

La sociedad contemporánea es conocida hoy en día como sociedad de la información, esto se debe en gran medida a la apertura de las comunicaciones y de la información derivada de las tecnologías; Crovi (2016) comenta que la tecnología ha generado procesos de cambio que tienen efectos desde perspectivas diversas: política, economía, filosofía, comunicación, sociología, entre otras.

Esto ha llevado a que entre las características del mundo actual sobresalga la fuerte competitividad de las organizaciones, esto obliga a que los países busquen aumentar las innovaciones como manera de elevar niveles de empleo y calidad de vida de la población. Haciendo a la información, el conocimiento y el aprendizaje como elementos esenciales en la actualidad.

Es por ello que como menciona Crovi (2016) existen varios nombres que designan al fenómeno actual: Informatización de la sociedad (Nora y Minc), sociedad de la comunicación (Vattimo), Revolución informacional (Miége), Informacionalismo (Castells), era de la postinformación (Negroponte), Sociedad del conocimiento (Drucker, Levy) o tercer entorno (Echeverría).

De manera general se entiende que la sociedad se ha vuelto más comunicacional que atraviesa todas las actividades, desarrollada de la mano de la globalización, cuya principal meta es la de acelerar la instauración de un mercado mundial abierto y autorregulado.

La información y el conocimiento se han convertido en un activo estratégico, que si es bien gestionado representará una ventaja competitiva para la organización, incluso puede llegar a representar mayor valor que el producto o servicios que se ofrecen.

Como lo indica Rodríguez (2012) las compañías de éxito son aquellas capaces de recoger sensiblemente la información interna y externa para crear de forma consistente y continuada nuevo conocimiento. A su vez, este conocimiento debe ser extendido a lo largo de la organización, para que se vea incluido en nuevos productos y servicios o características y funcionalidades de estos. Si se observa la siguiente imagen:

Figura 2. De los datos al conocimiento.

\begin{tabular}{|c|c|c|c|}
\hline & Datos & Información & ocimiento \\
\hline Contenidos & Eventos & Tendencias & Experto \\
\hline Forma & Transacciones & Patrones & Aprendizajes \\
\hline $\begin{array}{l}\text { Tareas de } \\
\text { información }\end{array}$ & Representación & Manipulación & Codificación \\
\hline Elemento humano & Observación & Juicio & Experiencia \\
\hline $\begin{array}{c}\text { Proposito } \\
\text { organizativo }\end{array}$ & Automatización & $\begin{array}{c}\text { Toma de } \\
\text { decisiones }\end{array}$ & Acción \\
\hline Valor & Elementos & $\begin{array}{l}\text { Reducción } \\
\text { de incertidumbre }\end{array}$ & $\begin{array}{c}\text { Nuevo } \\
\text { entendimiento }\end{array}$ \\
\hline
\end{tabular}

Fuente: Every Business is an Information Business, 2010

La forma en cómo se registran los eventos ligados a una transacción es mediante los datos, que pueden ser no solo observados sino incluso monitoreados, por ejemplo los asientos contables, una venta en un comercio, el desempeño docente o administrativo, etc. y la información es el resultado de interpretar dichos datos, mediante la manipulación y a partir del cual se establece un juicio y se toman decisiones.

De modo que el proceso continuo de toma de decisiones genera conocimiento, que es la experiencia, aprendizaje, entendimiento $\mathrm{o}$ juicio experto para intervenir en situaciones del futuro, elaborada a partir de información objetiva y del juicio experto.

\subsection{Intervención de la tecnología en la toma de decisiones}


Sáez Vacas (1987) señala que las tecnologías de la información son las que se aplican en la adquisición, procesamiento, almacenamiento y diseminación de información vocal, icónica, textual o numérica. De manera que la tecnología de la información es una herramienta indispensable cuando se trata de la recopilación, procesamiento, transmisión y almacenamiento de datos, mejorando el acceso a la información, lo que promueve cambios tanto en estructura como en la forma de tomar decisiones en la organización,

La tecnología ha formado parte del día a día de las organizaciones, sin embargo, con los avances en tecnologías de la información y comunicación, los alcances que tiene en la actualidad son mayúsculos, el mundo actual se encuentra hiperconectado.

Las organizaciones se encuentran abrumadas de datos que cada vez resulta más difícil comprender en su totalidad, según la empresa BSA, los datos tienen valor solo cuando son comprensibles; de otra forma, no son más que un montón de observaciones aleatorias.

Por lo que entre los principales pasos de la tecnología en la toma de decisiones es la recopilación de datos más importantes, eliminando espacios de incertidumbre y arbitrariedad, algunas de las incidencias que tiene en la actualidad la tecnología de la información en departamentos clave de cualquier organización son:

\subsubsection{Aplicación de las Tecnologías de Información en Operaciones}

Responsable de las líneas de producción, por lo que es evidente que se implementen sistemas de automatización, que conllevan a una delegación en la toma de decisiones, para Fernández et al. (2003) el aplicar las modernas tecnologías de información en procesos productivos, se pueden alcanzar simultáneamente objetivos de eficiencia y flexibilidad, dando lugar así, a la automatización. Cabe señalar que a través de la automatización (incorporación de la tecnología a los procesos de producción de manera autónoma).
Se logra una gestión integral que contribuye en la transformación del funcionamiento de la propia empresa en cuanto a transporte, manejo de materiales, control de inventarios, control de calidad, y mantenimiento de equipos e instalaciones, entre otras muchas aplicaciones autónomas en donde la tecnología se incorpora para reducir tiempos, movimientos y toma de decisiones por parte de la directiva del departamento operativo de la empresa.

\subsubsection{Aplicación de las Tecnologías de Información en RRHH}

Las actividades propias de este departamento cada vez se vuelven más complejas, su ámbito de competencia abarca temas de capacitación, selección de personal, temas de nómina, entre otras que mediante la tecnología de información se ha logrado que la toma de decisiones pueda dejar de estar centralizada.

Daft (2010) señala que las tecnologías en este departamento abarcan sistemas de información, de apoyo en la toma de decisiones y sistemas de información ejecutiva que facilitan la toma de decisiones rápida y efectiva, al integrar una gran cantidad de información que se manera en la organización, apoyando estos a los mandos intermedios y altos.

El uso de sistemas computacionales en el área de RRHH favorecen el uso de recursos, incluyendo tiempos y movimientos, propiciando eficiencias a sus actividades, gracias a que la tecnología incrementa la capacidad de procesamiento de la información y el aumento en la capacidad de respuesta.

\subsubsection{Aplicación de Tecnologías de la Información en Finanzas}

Uno de los temas más relevantes para el consejo de administración, accionistas o dueño de la empresa es el tema de la administración financiera, pues a partir de ahí se capitalizan las actividades propias de la empresa, favoreciendo su solvencia para responder a los embates económicos que puede llegar a presentar el propio ambiente político, económico, tributario y financiero del país. 
Diez (2008) comenta que, el uso de las TIC en finanzas, ha cambiado la forma en la que se registra y se llevan a cabo las tareas operativas mediante los instrumentos y programas, caracterizándolos por ser sistemas de información donde la disponibilidad de los datos es oportuna y veraz para la toma de decisiones.

La tecnología en este departamento contribuye a la eficiencia de los métodos utilizados en funciones como contabilidad, finanzas, entre otros al facilitar el logro de los objetivos de forma más rápida, favoreciendo el trabajo en equipo, y en materia de toma de decisiones.

Cohen et al. (2000) comenta que la tecnología ha mejorado de manera radical la eficiencia de las finanzas, al favorecer la toma de decisiones mediante el uso correcto de recursos disponibles para así obtener resultados deseados, tales como el procesamiento de órdenes de venta, cobros, cuentas por pagar, control de inventarios, órdenes de compra, contabilidad general y conciliaciones bancarias.

Los sistemas tecnológicos de base de datos, contratos inteligentes, block chain, instituciones de tecnologías financieras, y sistemas de pago vía internet también forman parte de los sistemas tecnológico que contribuyen a la efectividad y eficiencia de los métodos empleados por las organizaciones, así como al logro de objetivos organizacionales de manera rápida, facilitando la toma de decisiones.

Por su parte, Fonseca (2012) señala que los beneficios recibidos son múltiples, ya que muchas de las empresas beneficiarias han logrado incrementos en sus ventas, $\mathrm{y}$ disminuciones en sus costos de operación, reflejados en una mejor rentabilidad de sus negocios, menos errores sistemáticos, mejor manejo de los recursos, planeación de presupuestos y aumento en la competitividad.

Tanto el auge de las telecomunicaciones ha propiciado que las empresas conciban al factor tecnológico como un arma competitiva, debido a la rapidez en dar respuesta a las necesidades y requerimientos de mercado, mediante la toma de decisiones en base a información que se va registrando en bases de datos especializadas en la clasificación y selección de información propicia para ello, es importante analizar estas nuevas tendencias tecnológicas, aún consideradas como innovadoras que fomentan la mejora en las tomas de decisiones organizacionales.

\subsection{El Business Intelligence, Big Data y los Smart Contract}

La Inteligencia de Negocios es una herramienta que proporciona no solo información, sino también conocimiento de forma rápida, óptima y eficiente para sus usuarios, Baglietto (2018) define a la Inteligencia de Negocios como la habilidad empresarial para acceder y explorar información, con objeto de analizar la misma para desarrollar nuevas perspectivas y conocimientos que ayuden a mejorar el proceso de toma de decisiones.

Mientras que Asto Huamán (2018), señala que el Business Intelligence es la habilidad para transformar los datos en información, y la información en conocimiento, de forma que se pueda optimizar el proceso de toma de decisiones.

Cordero Cerda (2017) comenta que entre las ventajas que aporta las herramientas de la Inteligencia de Negocios en las organizaciones destacan:

a) Control y reducción de gastos mediante el seguimiento presupuestario y el uso de la analítica.

b) Mayor competitividad $y$ posicionamiento, gracias a una buena gestión y retención del talento.

c) Mejora en la gestión organizacional, por mediciones de evaluación, seguimiento del RRHH y la calidad en el proceso operativo.

d) Mayor eficiencia en sus procesos.

Méndez del Río (2016) define la Inteligencia de Negocios como un conjunto de herramientas y aplicaciones para ayudar a la toma de decisiones que posibilitan acceso interactivo, análisis y manipulación de información corporativa de misión crítica, aportando un conocimiento valioso sobre la información operativa identificando problemas y oportunidades de negocio. 
Así es como la sociedad de la información ha propiciado la necesidad de contar con mejoras, más rápidos y más eficientes métodos para recopilar y transformar datos de una organización, la Inteligencia de Negocios representa entonces el conjunto de metodologías, aplicaciones, prácticas y capacidades enfocadas a la creación y administración de información que permite tomar mejores decisiones a los usuarios de la organización.

Curto Díaz (2016) comenta que existen situaciones en las que la implantación de un sistema de inteligencia de negocios resulta adecuada, destacando entre otras:

Figura 3. Escenarios en donde resulta adecuada la implementación de un sistema de Inteligencia de Negocios.

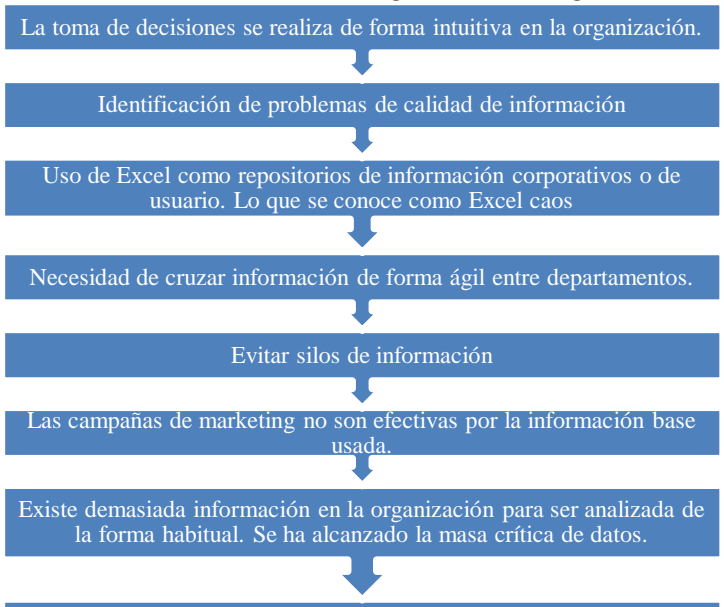

Es necesario automatizar los procesos de extracción y distribución de

Fuente: Curto Díaz, 2016.

Las tecnologías y factores de la inteligencia de negocios logran colocar datos al alcance de los responsables de la toma de decisiones, empleando herramientas que extraen datos de la mejor manera para después almacenarlos en un repositorio optimizando la entrega de información de forma rápida y resumida que haga posible su análisis reduciendo el tiempo en que se formula la información e incrementando el tiempo invertido en su análisis para la debida toma de decisiones.

Otra de las herramientas tecnológicas que coadyuvan con la toma de decisiones es el denominado Big Data, que son activos de información de gran volumen o variedad que permiten formas innovadoras de procesamiento de información, permitiendo mayor comprensión al momento de la toma de decisiones y la automatización de procesos, este almacenamiento masivo tiene potencial de mejorar la eficiencia interna y las operaciones mediante la automatización robótica de procesos, logrando analizar de manera inmediata e integrarse en procesos comerciales con el objetivo de efectuar tomas de decisiones automatizadas, además de facilitar la evaluación de escenarios en tiempo real, generando valor en las decisiones empresariales.

Otra de las innovaciones tecnológicas que está en pleno auge y que facilita la toma de decisiones en las organizaciones son los denominados contratos inteligentes o Smart Contracts, de los cuáles, Valencia Ramírez (2019) señala que:

"El contenido de un contrato inteligente se centra a establecer una limitación que recae sobre las distintas interpretaciones que pudieran tener el contenido de lo estipulado, ya que este se configura como un protocolo informático susceptible de hacer realidad lo pactado y nada más que lo pactado, al contrario de lo que puede ocurrir con contratos electrónicos o los tradicionales, en los que surgen disputas sobre su contenido e interpretación de éstos".

Que son secuencias de código y datos que efectúa la operación para la cual fueron programados, como consecuencia no son contratos en términos jurídicos, ya que la conceptualización jurídica sobre un contrato inteligente sería la de un programa informático que trabaja en base a una serie de instrucciones autoejecutables codificadas, donde el código mantiene aquellas que tengan relación al cumplimiento de las cláusulas y en donde existe acuerdo de voluntades, concurriendo así su relación en el mundo legal, y que es importante señalar, no pueden ser manipuladas, de modo que son:

1. Inmutable. - una vez creado el Smart Contract no puede ser modificado de nuevo por lo que nadie puede modificarlo $\mathrm{y}$ 
cambiar las normas o acuerdos dispuestos anteriormente.

2.- Descentralizada. - Todos deberán validar ese contrato en la red, por lo que una sola persona no puede mandar sobre el resto ni actuar por su cuenta.

Estos contratos tienen múltiples usos, por ejemplo, los bancos pueden usarlos para dar préstamos u ofrecer pagos automáticos, las compañías de seguros para procesar reclamaciones y las empresas de mensajería para pagos en las entregas, por lo que simplifican la toma de decisiones comerciales a través de contratos codificados.

\section{METODO DE INVESTIGACIÓN.}

Con el objetivo de dar sustento a los criterios que caracterizan al presente estudio, se recurrió al tipo descriptivo, que según Hernández Sampieri (2010) busca especificar las propiedades, características y perfiles de personas, grupos, comunidades, procesos, objetos o cualquier otro fenómeno que se someta a un análisis.

El estudio se centra en señalar las implicaciones que tiene la incorporación de la tecnología en la toma de decisiones, situación que hasta el momento, ha quedado fundamentada a través de la técnica documental, recopilada a través de la consulta de libros, publicaciones en revistas especializadas, y estudios académicos que sirven de fundamento documental para señalar las implicaciones que está presentando el uso de diferentes tecnologías en los diferentes momentos de toma de decisiones que se realizan, no solo en la alta dirección, sino incluso en departamentos de producción y RRHH.

Este estudio solo pretende recoger información sobre los conceptos o variables a que se refieren, como lo es la tecnología y la toma de decisiones empresariales, de modo que el objetivo no es indicar como se relacionan, sino realizar la investigación documental. De manera que la presente investigación es de corte documental, obtienen información mediante la recopilación, organización y análisis de fuentes documentales escritas con el objetivo de ser analizados y de esta manera aportar conocimiento sobre el tema de la tecnología y sus implicaciones en la toma de decisiones empresariales, mediante el uso de una metodología cualitativa, debido a que la acción indagatoria que se realiza, tiene un dinamismo entre los hechos y su interpretación, utilizando la recolección y análisis de datos para afinar una serie de resultados y conclusiones señaladas al final de la investigación, finalmente es una investigación deductiva.

\section{RESULTADOS}

\subsection{La tecnología en la toma de decisiones como ventaja competitiva sostenible en la empresa}

Toda vez que se han señalado las bases conceptuales y contextuales de la tecnología en la toma de decisiones, se puede señalar que esta relación es jerárquica para conseguir una ventaja competitiva en la industria en la que se encuentre la empresa, sin duda las grandes empresas manejan sistemas de toma de decisiones sistematizadas y automatizadas con el objetivo no solo de simplificar la toma de decisiones, sino también para minimizar los costos que implica el tiempo invertido en estos escenarios.

Sin embargo, empresas medianas y pequeñas se enfrentan a ciertos obstáculos en esta materia, ya sea en el acceso a la información que tiene la persona que decide a la hora de la toma de decisiones, así como en las aplicaciones informáticas utilizadas en el flujo de información, en donde se pueden ocasionar dificultades en el mismo.

Derivado de la investigación documental, se ha podido vislumbrar la importancia de la relación que existe entre la comunicación, el manejo de la información y la adopción de las TIC con la mejora administrativa y toma de decisiones reside en el hecho de la facilidad de visualizar los datos y relaciones que existen entre ellos, incrementando la probabilidad de tomar decisiones asertivas, a pesar de esto, no solo se trata de adquirir la tecnología, sino de hacer un uso correcto de las mismas para poder optimizar la toma de decisiones y asegurar que el futuro de la empresa se encuentre bien direccionada. 
Esto con el objetivo de generar una ventaja competitiva sostenible para la empresa a través de hacer un uso de la tecnología en la toma de decisiones, ya que decidir supone la mejor opción entre una serie de posibilidades, partiendo de la identificación de une escenario problemático o no, durante el cual será necesario la examinación de las diferentes alternativas, aplicando la más adecuada para posteriormente analizar si se ha conseguido los objetivos planeados.

Por ello, el administrador debe estar consciente de los principales errores y sesgos que se producen durante el proceso de toma de decisiones perjudicando la obtención de decisiones idóneas en cada situación, mediante la adquisición de habilidades precisas para resolver situaciones conflictivas que derivan de las tensiones que se producen en el proceso de toma de decisiones sensibles en el aspecto personal y social de la empresa.

En los comercios minoristas, el uso de las tecnologías para la toma de decisiones permite la reducción de costos de operación, que se derivan de la utilización de menos tiempo en cada transacción con el cliente, proveedores y trabajadores, además de acercar al empresario a una optimización del almacenaje y manejo de existencias mínimas por la entrega a tiempo, lo que agiliza la cadena de producción y distribución, lo que representará una diferenciación en la operatividad, convirtiéndose en ventajas competitivas mediante tomas de decisiones certeras.

Y para ello, el diseño de sistemas estacionarios, que contemplen tecnologías, desde las más sencillas como el manejo de bases de datos con información de uso cotidiano, hasta sistemas más complejos de Big Data en donde la programación permita la automatización y sistematización de la información, de modo que se tomen decisiones de manera efectiva.

\subsection{La estandarización, sistematización y automatización de la toma de decisiones en las empresas a través de la implementación de tecnología}

La estandarización de la toma de decisiones tiene el objeto de unificar los resultados en las organizaciones que utilizan diferentes prácticas en un mismo proceso; sobre este concepto, Martínez (2015) señala que es el proceso de elaborar, emplear y optimizar las reglas que se aplican a distintas actividades con la finalidad de concretarlas y mejorarlas, de manera que va a ser un mecanismo que coadyuve en la coordinación, cabe aclarar que la empresa desarrolla su actividad en entornos cambiantes e impredecibles.

Ya sean consecuencia de decisiones de clientes, la complejidad del producto, la dificultad de planificación normalmente da pie a la necesidad de agilizar la respuesta ante ciertos problemas, la estandarización en la gestión de la toma de decisiones proporciona en estos casos información sobre pautas a seguir, Vázquez (2015) comenta que:

El valor operativo más interesante de la estandarización es la creación de una disciplina que permite documentar todas las acciones puestas en práctica y ciertos datos fundamentales, asegurando la creación de una estructura con la que se puede obtener información y estandarizar los procesos.

La sistematización es más un proceso de aprendizaje y construcción de conocimiento desde la práctica, Dávila (2016) señala que es un proceso de auto reflexión, donde los mismos actores, identifican las grandes lecciones, en un marco de enseñanza aprendizaje, es decir, la sistematización se produce una vez que se ha generado conocimiento, en base a la información que ha sido estructurada y estandarizada.

La sistematización construye conocimiento, los grupos en su conjunto generan ambientes de análisis y reflexión sobre diferentes escenarios, identificando cuáles han sido los más relevantes para el equipo y así genera nuevo aprendizaje, sin cambiar concepciones personales, ni creencias.

Finalmente, la automatización viene a dar una innovación tecnológica tanto a la estandarización como a la sistematización, ya que da agilidad al procesamiento de la información, de manera que las ventajas para la empresa en materia de transformación 
digital van encaminadas a la automatización de procesos.

Según Cervantes (2017) ésta es la sustitución de tareas en las que hay un alto porcentaje de intervención humana por otras tareas que pueden ser desempeñadas de forma automática por plataformas software adaptadas a tal efecto, y para ello se ocupan mecanismos como:

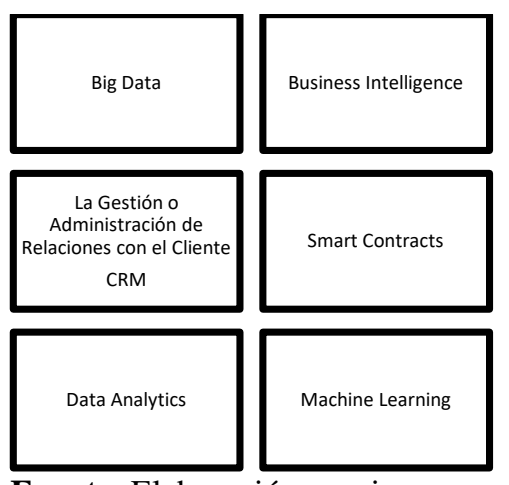

Fuente: Elaboración propia.

Las ventajas de la automatización son variadas, desde el primer momento de su implementación se simplifican tareas, se reducen riesgos en el error humano, aumento de la calidad, precisión, exactitud y velocidad, así como de una reducción significativa de la variabilidad y del costo de los procesos.

4.3. Retos y oportunidades de la implementación de la tecnología para la toma de decisiones en las organizaciones

Las organizaciones son consideradas como "inteligentes" en medida que logren hacer uso de su información para crear innovaciones y conocimiento que coadyuven en la mejora continua de su toma de decisiones, reduciendo tiempos de respuesta para evitar la pérdida de oportunidades y así incrementar la rentabilidad de la empresa.

Como consecuencia, la implementación de sistemas de comunicación e información que fluyan de manera constante de manera vertical, horizontal y transversal, juegan un papel fundamental en cualquier organización, pues ésta puede facilitar todo tipo de tareas y con esto aportar de manera colectiva ventaja competitiva a lo largo de la cadena de producción o de servicio, a través del uso de tecnologías de información que permitan agilizar los procesos y la toma de decisiones.

La tecnología ha demostrado un avance acelerado y una integración con las diferentes industrias comerciales, tal es el caso de las instituciones de tecnología financiera, sistemas que han revolucionado la manera de captar y colocar financiamiento a través de medios electrónicos, en donde se utilizan sistemas programados y automatizados en donde se responde de manera automática a las decisiones de cada uno de los usuarios.

La tecnología es parte ya de la vida diaria del ser humano, por lo que debe ser igual con las organizaciones, no limitándose a las de mayor tamaño, pues incluso las pequeñas empresas no pueden rezagarse en el uso de correos electrónicos, fax, teléfono, manejo de sitios web o intranet, o sistemas CMR que si bien están orientados a gestionar información para la toma de decisiones, también es cierto que esta metodología automatizada está quedando rezagada debido a sistemas de inteligencia de negocios cuyos alcances y beneficios se extienden más allá de una simple base de datos.

\section{CONCLUSIONES}

La toma de decisiones es diaria y se extiende a todas las áreas de la organización, con una evidente dependencia de información como recurso estratégico, y mientras mayor sea la responsabilidad jerárquica del trabajador, mayor será el grado de consecuencias que tendrá una mala toma de decisiones, por lo que no se requieren solo de programas o procedimientos que aminoren la incertidumbre, sino que se requiere de tecnología que brinde competencias, dinamismo y capacidad organizacional para una acertada toma de decisiones estratégica, obteniendo con esto ventaja competitiva en un mercado globalizado.

Sin duda, la selección y administración apropiada de sistemas de información resulta ser un desafío esencial para los responsables de esta tarea, pues representa un medio que pudiera dinamizar las operaciones administrativas, e incluso operacionales de la organización, productividad, servicio y satisfacción del cliente. 
Por ello los responsables de la toma de decisiones deben comprender que la información no es solamente un producto de la conducción empresarial, sino que es un combustible que se alimenta de los propios negocios y que, si no se nutre y se gestiona a través de la tecnología correspondiente, puede representar un factor crítico para la terminación del fracaso en una empresa. 


\section{REFERENCIAS}

Asto Huamán, L. (2018). INTELIGENCIA DE NEGOCIOS EN LA GESTIÓN ACADÉMICA DE LA EDUCACIÓN SUPERIOR UNIVERSITARIA. Perú: Universidad Nacional del Altiplano.

Banglietto, A. (2018). Hacia una economía del conocimiento. Madrid, España: PricewaterhouseCoopers.

BSA. (2018). ¿Por qué son tan importantes los datos? México: 2018.

Castillo Hernández, M. (2018). Toma de Decisiones en las empresas. Colombia: Uniandes.

Cohen, K., Asin, D., \& Lares, E. (2000). Sistemas de Información para los negocios. Un enfoque de toma de decisiones. México: McGraw Hill.

Cohen, M. (2002). Algunos aspectos de uso de la información en la economía de la información. Brasil: Ciencias de la Información.

Cordero, R. O. (2017). La utilización de Business Intelligence como propuesta para mejorar los indicadores de deserción de los estudiantes de laEscuela de Ingeniería Informática. Chile: PONTIFICIA UNIVERSIDAD CATÓLICA DE VALPARAÍSO.

Crovi, D. (2016). Sociedad de la Información y el conocimeinto. Entre el optimismo y la desesperanza. México: Universidad Nacional Autónoma de México.

Curto, J. (2016). Introducción al Business Intelligence. Barcelona, España: UOC.

Daft, R. (2010). Teoría y diseño de la Organización. México: Thomson Editores.

Davenport, T. H., \& Prusak, L. (1998). Working Knowledge: how organisations manage what they know. Boston: Harvard University Press.

Dávila, R. (2016). Sistematización del Proceso. Guatemala: Instituto Interamericano de Cooperación.

Díez, T. (2008). Sistemas de Información para la nueva reforma contable. México: Unirroja.

Earl, M. (2010). Every Business is an Information Business. Londres, Inglaterra: Financial Times Prentice Hall.

Fernández, E., Avella, L., \& Fernández, M. (2003). Estrategia de producción. España: McGraw Hill. Fonseca, D. (2012). La prospectiva y el conocimeinto de las TIC en las pymes del departamento de Boyacá. Colombia: Pensamiento y Gestión.

Hernández, R. (2016). Metodología de la Investigación. México: McGraw Hill.

Hodge, B., William, A., \& Lawrence, G. (1998). Teoría de la Organización. Un Enfoque Estratégico. Madrid, España: Prentice Hall.

Juma, N. (2004). Intellecual capital and performance of new venture hight tech firms. EEUU: International journal of innovation and magnament.

Martínez, A. (2015). Gestión por procesos de negocio. Madrid, España: Ecobook.

Mendez del Río, L. (2016). Más allá del Business Intelligence. España: Gestión 2000.

Molina, J. L. (2015). La Gestión del Conocimiento en las Organizaciones (4ta ed.). México: Libros en Red.com.

Nagles, N. (2015). La Gestión del Conocimiento como fuente de Innovación. Bogotá, Colombia: Revista Escuela de Administración de Negocios.

Peñaloza, M. (2010). Teoría de las decisiones perspectivas. Cochabanmba , Bolivia: Universidad Católica Boliviana San Pablo.

Reyez, L. A. (2014). Tecnología y capital intelectual comop parte del proceso productivo en empresas de la ciudad de Sucre. Bolivia: Universidad Mayor Real.

Rodríguez, J. R. (2012). Gestión de la infromación y el conocimiento. Catalunya, España: UOC.

Sáez, F. (1987). Towards a conceptual remodeling of information technologies based on a broad consideration of complexity. EEUU: 31st Annual Meeting of the International Society for General Systems Research.

Valencia, J. P. (2019). “Contratos Inteligentes”. México: Editorial SEICIT.

Vázquez, C. (2015). Calidad y estandarización como estrategias competitivas en el sector agroalimentario. Maracaibo, Venezuela: Universidad del Zulia. 